\title{
A Knowledge Management Framework of the Information Technology-Based Approach for Improving Occupational Safety Training of Young Workers
}

\author{
Irena Hejduk \\ Warsaw School of Economics, Warsaw, Poland \\ Waldemar Karwowski \\ University of Central Florida, Orlando, USA
}

\begin{abstract}
This paper aims to develop a novel knowledge management framework for improving the system of pro-safety attitudes among young worker population in Poland. This is done through an efficient use of existing sources of explicit and tacit knowledge in the field of vocational occupational safety and health (OSH) training. This project identifies three specific research steps for the development of the knowledge management framework of the information technology-based approach for improving occupational safety training of young workers, including: (1) the development of the conceptual model of the flow of explicit knowledge of OSH; (2) evaluation of the effectiveness of formal and explicit knowledge sources for OSH and training methods using these sources in the context of attitudes towards health and safety at work; and (3) verification of the proposed model of vocational training with the use of combined explicit and hidden knowledge. The proposed framework includes consideration of the effectiveness of the formal and informal sources of safety knowledge. A mapping from the formal sources of explicit knowledge about the occupational safety to expected attitudes (hidden knowledge) towards OSH outcomes, including emotional, cognitive, and behavioral aspects has been used. The above framework should help to improve the system of vocational training for young workers in Poland.
\end{abstract}

Keywords: knowledge management, occupational safety, education and training, young workers

\section{Introduction}

The survival and competitiveness of EU enterprises and their future expansion depend on the commitment of human resources, functioning in a high quality working environment that provides both safe and healthy conditions. According to Organization for Economic Co-operation and Development [OECD] (2014), the report "OECD Employment Outlook 2014" ranks Poland in 26th place with respect to the level of performance in all major categories affecting the quality of jobs, such as the level of earnings, inequality in wages, the risk of losing a job and being unemployed, the level of benefits for the unemployed, and working comfort. Changes in work organization resulting from advances in information technology, especially the achievements of widely

Irena Hejduk, Ph.D., professor, Head of Management Systems Department, Warsaw School of Economics, Warsaw, Poland. Waldemar Karwowski, professor, Chair of IEMS Department, University of Central Florida, Orlando, Florida, USA.

Correspondence concerning this article should be addressed to Irena Hejduk, Warsaw School of Economics, Aleje Niepodległości 162, Warsaw 02-554, Poland. 
accessible mobile communication networks, open huge opportunities for flexible and interactive processes at the workplace across EU.

There has been a growing diversity of the labor force, as reflected by atypical contractual arrangements and patterns of work, as well as a higher level of job turnover associated with shorter terms of employment, especially for young workers. The recommendation of the Council of the European Union for the Polish economy (2012-2016) was to limit temporary contracts because of its negative impact on the quality of human capital, its performance, and fewer opportunities afforded to workers to participate in vocational training. It was also recommended to increase the cooperation between schools and employers, as well as improve the quality of vocational education.

The potential impact of information technology on human work in general and occupational safety and health (OSH) in particular, can be considered from the perspective of three different viewpoints. The first perspective relates to the changes in terms of how employees perform their jobs; the second is the increase in the use of existing information technologies at the workplace; and the third perspective is the development and evolution of the way in which information technology allows businesses to organize work in general.

The primary objective of the current research was to develop a novel knowledge management framework for improving the system of pro-safety attitudes among young workers, in particular through an efficient use of existing sources of explicit and tacit knowledge in the field of vocational OSH training. This project also aims to identify three specific research steps for the development of the knowledge management framework of the information technology-based approach for improving occupational safety training of young workers, including the followings:

- development of the conceptual model of the flow of explicit knowledge of OSH in the system of vocational training for young workers;

- evaluation of the effectiveness of formal and explicit knowledge sources for OSH, and training methods using these sources in the context of attitudes towards health and safety at work exhibited by the young workers;

- verification of the proposed model of vocational training with the use of combined explicit and hidden knowledge.

The above frameworks should help to improve the system of vocational training for young workers in EU, in particular through the effective use of combination of explicit and hidden OSH knowledge.

\section{Literature Review}

This project is focused on improving the system of pro-safety attitudes among young workers through an efficient use of existing sources of explicit and tacit knowledge. The currently used system is based largely on the transfer of explicit knowledge presented in terms of procedures or instructions (Takeuchi \& Nonaka, 2000). Such knowledge is often difficult to verbalize and apply in the context of work, which makes it difficult to acquire and codify (Polanyi, 1983; Takeuchi \& Nonaka, 2000). However, the use of tacit knowledge in the field of occupational health and safety has been advocated to have a positive effect on the operation of enterprises (Sherehiy \& Karwowski, 2006; Schulte, Lentz, Anderson, \& Lamborg, 2004).

The significance of tacit knowledge and its elements for the development of safety culture has been confirmed by many survey studies, according to which tacit knowledge is complementary to explicit knowledge (Mascini \& Bacharias, 2008; Moulton \& Forrest, 2005). This is because the formal regulations do 
not account for all risky situations, and often the proposed procedures are not always the best in relation to a given workplace condition.

The new EU strategic framework in the field of health and safety (2014-2020) notes that the information technology offers new possibilities in the field of flexible work and interactive work processes. As a result, there is a greater variety of the available work force and a higher level of job rotation. This leads to a greater uncertainty in terms of employment and, consequently, higher levels of work-related stresses. The recent Eurobarometer survey shows that the majority of respondents $(53 \%)$ consider work-related stress as the most important threat to their professional well-being. Repetitive motions or uncomfortable postures on the job are indicated by $28 \%$ of respondents and lifting or carrying of heavy loads by $24 \%$.

Many physical hazards to workers' health are subject to the provisions of the EU labour code, for example, the employers in accordance with Act. 227 of the labor code are obliged to take measures to prevent occupational diseases and other diseases associated with workplaces, and are required to introduce and maintain efficient work-aiding devices that reduce or eliminate factors harmful to health and measure their effects. This is especially important to environments in which workers are exposed to chemicals that are harmful to their health. The quoted statistics also indicate the presence of risks to employees' mental health.

Given that good health is vital for both a stable life and active aging, more and more attention is paid in EU to stress management and assessment in terms of psychosocial risks in the workplace. The causes of psychosocial risks include incorrect planning, organization, and management of the workplace, as well as low levels of safety culture. In order to reduce the effects of occupational stress and psychosocial risks, the EU countries promote development of the healthy and safe workplaces, especially at the level of small and medium-sized enterprises. According to EU strategic framework for OSH (2014-2020), the small and medium-sized enterprises are more difficult to operate in compliance with all safety requirements. Therefore, it is necessary to provide both financial support and technical assistance to such companies through implementation of such tools as online interactive risk assessment (OiRA).

The OiRA system has been created in order to provide easy way to use decision-support tools that guide the micro and small organizations through the process of risk assessment. The OiRA software, developed by EU-OSHA in 2009 is based on the Dutch risk assessment tool called RI \& E. It is expected that application of OiRA will achieve an equivalent level of success in other EU countries. The new OiRA applications include a variety of service sectors, including hairdressing, catering, and private security. Similar applications have been developed in other countries, including Belgium, Spain, Greece, and Lithuania in cooperation with the social partners of the EU. This represents a step forward in the direction of placing OiRA in the center of EU risk prevention strategies. It is noted that such an approach should also include educational activities aimed to increase awareness of the importance of health and safety at work, as well as their impact of occupational safety on the quality of human capital.

\section{Research Methods}

At the first stage of the project, the English and Polish-language literature devoted to knowledge management and training of young workers in OSH was reviewed. Such literature was then subjected to meta-analysis, consisting of a critical analysis of the published results. Next, comprehensive research questionnaires have been developed to study the current state of knowledge of young workers (defined as those who worked less than three years) employed in industries with high and low levels of occupational safety. 


\section{Results}

The following safety knowledge questionnaire was adopted (see Table 1) based on the synthesis of several validated studies (Vinodkumar \& Bhasi, 2010). This questionnaire will be used at the second stage of the project that will investigate the effectiveness of mobile technology applications for the purpose of OSH training utilizing a large sample of young workers through a field study.

Table 1

Safety Questionnaire

Management commitment

1. Safety is given high priority by the management.

2. Safety rules and procedures are strictly followed by the management.

3. Corrective action is always taken when the management is told about unsafe practices.

4. In my workplace, managers/supervisors do not show interest in the safety of workers.

5. Management considers safety to be equally important as production.

6. I feel that management is willing to compromise on safety for increasing production.

7. When near-miss accidents are reported, my management acts quickly to solve the problems.

8. My company provides sufficient personal protective equipment for the workers.

Safety training

1. My company gives comprehensive training to the employees in workplace health and safety issues.

2. Newly recruits are trained adequately to learn safety rules and procedures.

3. Safety issues are given high priority in training programmes.

4. Management encourages the workers to attend safety training programmes.

5. Safety training given to me is adequate to enable me to assess hazards in workplace.

Workers' involvement

1. Management always welcomes opinion from employees before making final decisions on safety related matters.

2. My company has safety committees consisting of representatives of management and employees.

3. Management promotes employees involvement in safety related matters.

4. Management consults with employees regularly about workplace health and safety issues.

Safety communication and feedback

1. Management operates an open door policy on safety issues.

2. There is sufficient opportunity to discuss and deal with safety issues in meetings.

3. The target and goals for safety performance in my organization are not clear to the workers.

4. There are open communications about safety issues in this workplace.

Safety rules and procedures

1. The safety rules and procedures followed in my company are sufficient to prevent incidents occurring.

2. My supervisors and managers always try to enforce safe working procedures.

3. Safety inspections are carried out regularly.

4. The safety procedures and practices in this organization are useful and effective.

Safety promotion policies

1. In my company, safe conduct is considered as a positive factor for job promotions.

2. In my company, employees are rewarded for reporting safety hazards (thanked, cash or other rewards, recognition in newsletter, etc.).

3. In my company, safety week celebration and other safety promotional activities arranged by the management are very effective in creating safety awareness among the workers.

4. There exists very healthy competition among the employees to find out and report unsafe condition and acts. 
Table 1 to be continued

Safety knowledge

1. I know how to perform my job in a safe manner.

2. I know how to use safety equipment and standard work procedures.

3. I know how to maintain or improve workplace health and safety.

4. I know how to reduce the risk of accidents and incidents in the workplace.

5. I know what are the hazards associated with my jobs and the necessary precautions to be taken while doing my job.

Safety motivation

1. I feel that it is important to maintain safety at all times.

2. I believe that safety at workplace is a very important issue.

3. I feel that it is necessary to put efforts to reduce accidents and incidents at workplace.

4. I feel that it is important to encourage others to use safe practices.

5. I feel that it is important to promote safety programmes.

Safety compliance

1. I use all necessary safety equipments to do my job.

2. I carry out my work in a safe manner.

3. I follow correct safety rules and procedures while carrying out my job.

4. I ensure the highest levels of safety when I carry out my job.

Safety participation

1. I always point out to the management if any safety related matters are noticed in my company.

2. I put extra effort to improve the safety of the workplace.

3. I voluntarily carryout tasks or activities that help to improve workplace safety.

4. I encourage my co-workers to work safely.

Source: Vinodkumar and Bhasi (2010).

\section{Discussion}

The EU has been a leader in assuring the safe working conditions in the past and is expected to continue implementing systematic measures to promote the high standards of working conditions in order to minimize the level of accidents at work and occupational diseases. The EU member states have already implemented most of the legislative and non-legislative initiatives set out in the strategy for health and safety in the past (2007-2012). According to the Report on "Agenda for New Skills and Jobs", the proportion of young people taking up studies at universities has been steadily increasing and the working conditions and the quality of jobs have been improving.

The management of occupational health and safety in the EU can be considered in a broad context of social well-being and development. The first component of such a perspective is the competitiveness of the national economy based on the high quality of human capital. The second component is the cost savings associated with the avoidance of economic losses resulting from absenteeism, increased demand for health services, as well as premature withdrawal from the labor market. The third component is the physical and mental health of all citizens of the European Union, while the fourth component is a contribution to the development of safe work standards worldwide.

As estimated by the European Centre for the Development of Vocational Training (CEDEFOP), by 2020 there will be growing demand for highly skilled workers in Poland, with a decline in the demand for workers with low-skills. For this reason, it is necessary to develop work safety competencies through continuous 
employee education by adopting new and more effective directions of investment in OSH training, including:

- development of a system for identification of employee qualifications that have been acquired outside the formal educational system;

- implementation of a system for collection and transfer of best educational practices in OSH, consistent with the EU mandated system of national education qualifications.

This project promotes the application of mobile technology to educational process in line with the recommendations of the Council of the $\mathrm{EU}$, in particular with respect to the use of new technologies in educational processes, which should also be applied to improve working conditions and quality of occupational safety health training and education.

\section{Conclusions}

The state of knowledge of safety polices and best practices among the young employees in Poland vary widely. Currently, the available scientific knowledge is not effectively utilized in the design and implementation of comprehensive training solutions to ensure safety of young workers in industry. The aim of the first phase of this project was to develop a conceptual framework and a model of the flow of knowledge focused on improving the system of vocational training for young workers, with due considerations of organizational and safety cultures.

The expected benefits of this project will be an improvement in the current system of vocational training for young workers. In particular, the emphasis will be on effective use of existing sources of explicit (formal) knowledge combined with the hidden knowledge represented by workers' attitudes and pro-safe behaviors in the context of a specific safety culture. It is expected that the results of this project will be implemented in at least 20 vocational schools educating 3,000 future employees. Within one year of the completion of the project, authors expected significant increase in the pro-safety behavior among young workers associated with the cooperating schools.

\section{References}

Mascini, P., \& Bacharias, Y. (2008). Formal and informal risk handling strategies: The importance of ethnographic research for safety surveys. Proceedings from the 4th International Working on Safety Network Conference, Crete.

Moulton, B., \& Forrest, Y. (2005). Accidents will happen: Safety-Critical knowledge and automated control systems. New Technology, Work and Employment, 20(2), 102-114.

Organization for Economic Co-operation and Development [OECD]. (2014). OECD employment outlook 2014. Paris: OECD Publishing.

Polanyi, M. (1983). Tacit dimension. London: Peter Smith Publications.

Schulte, P. A., Lentz, T. J., Anderson, V. P., \& Lamborg, A. D. (2004). The broader context: Knowledge management in occupational hygiene: The United States example. Annals of Occupational Hygiene, 48(7), 583-594.

Sherehiy, B., \& Karwowski, W. (2006). Knowledge management for occupational safety, health and ergonomics. Human Factors and Ergonomics in Manufacturing, 16(3), 309-320.

Takeuchi, H., \& Nonaka, I. (2000). Reflection on knowledge management from Japan. In D. Morey, M. T. Maybury, and B. M. Thuraisingham (Eds.), Knowledge management: Classic and contemporary works (pp. 183-186). Cambridge: The MIT Press.

Vinodkumar, M. N., \& Bhasi, M. (2010). Safety management practices and safety behaviour: Assessing the mediating role of safety knowledge and motivation. Accident Analysis and Prevention, 42, 2082-2093. 